\title{
Factors Leading to Acquired Bacterial Resistance Due to Antibiotics in Pakistan
}

\author{
Ali Mustufa*, Irtiqa Ahmed, Mahrukh Fareed and Tooba Anwar \\ Jinnah Sindh Medical University, Pakistan \\ Received: 阱 August 01, 2018; Published: 眥 August 09, 2018 \\ *Corresponding author: Ali Mustufa, Jinnah Sindh Medical University, Karachi, Pakistan
}

\begin{abstract}
Antibiotics are medicines used to prevent and treat bacterial infections. Antibiotic resistance occurs when bacteria change in response to the use of these medicines. Bacteria, not humans or animals, become antibiotic-resistant. Antibiotic resistance occurs when an antibiotic has lost its ability to effectively control or kill bacterial growth. Antibiotic resistance is a major concern of overuse of antibiotics. In other words bacteria are resistant and are able to multiply in the presence of therapeutic levels of an antibiotic. Antibiotic resistance has become a major issue in the $21^{\text {st }}$ century and is posing as a major blip on the danger radar. Pakistan being one of only three polio-suffering countries is also suffering from bacterial resistance caused by poor use of Antibiotics. Being a third world country, Pakistan suffers from avoidable causes of antibiotic resistance but lacks the basic necessities which provides favorable environment to bacteria and produce resistance to its killer/inhibitor. Following article focuses primarily on the factors leading to acquired bacterial resistance to Antibiotics in Pakistan.
\end{abstract}

\section{Introduction}

\section{The Capacity of Bacteria to Resist Against The Effects of an Antibiotic is Called Antibiotic Resistance}

This resistance is due to the change in nature of the bacteria and becomes severe due to several multiplications of bacterial generations which cause destruction in human body [1]. Another most common cause of antibiotic resistance in Pakistan is that the patients get antibiotics from medical stores without the physician's |description [2]. Antibiotic consumption in Pakistan between 2000 and 2015 increased 65\% from 800 million defined daily doses (DDD) to 1.3 billion DDD. The antibiotic consumption rate in the country witnessed a surge from 16.2 DDD to 19.6 DDD per 1,000 inhabitants per day [3]. Multidrug resistance (MDR) is increasing day by day in Pakistan [1]. A study found that Pakistan was the third highest consumer of antibiotics after India and China among the 76 low- and middle-income countries it surveyed [4] (Figure 1).

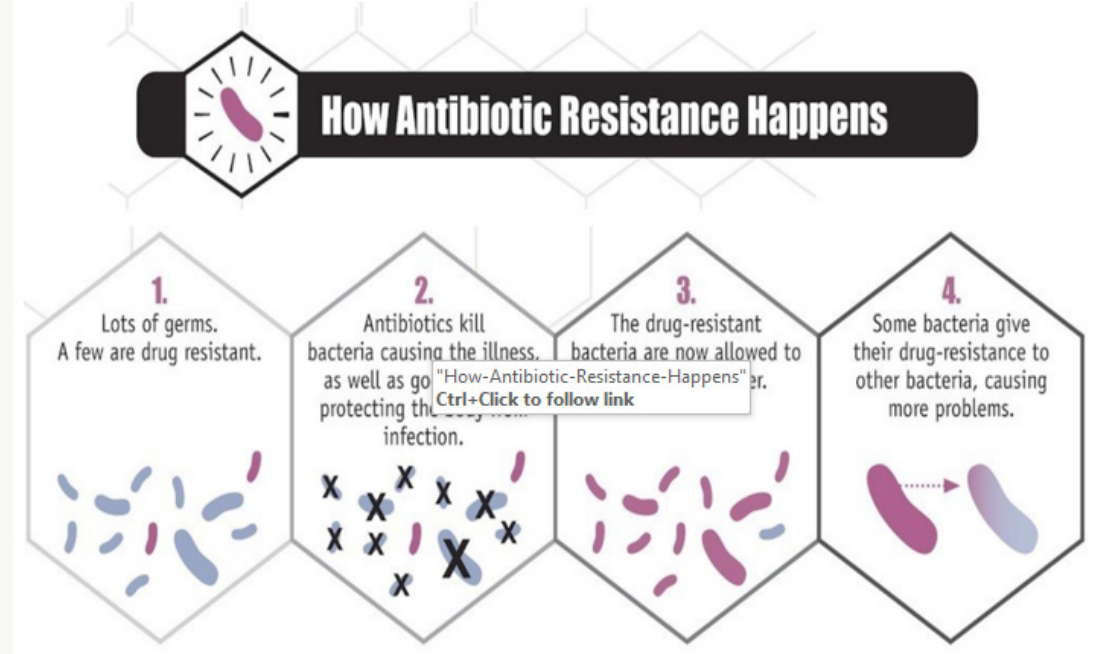

Figure 1: How Antibiotic Resistance Happens. 


\section{Misuse}

The prevalence of misuse of antibiotics is common in under developed $\backslash$ developing countries as compared to developed countries. Pakistan being a developing country is majorly suffering misuse of antibiotics as a result developing resistant to over the counter antibiotics and playing a part in the evolution of mutated genes with far more resistance than their precursor form of the micro-organism which not only is a problem for Pakistan but for the entire human \animal population. Below are discussed on the issue on causes of antibiotics misusage in Pakistan w.r.t it's socioeconomic and literacy status [5].

\section{Cheap and Easy Availability}

Pakistan is a poor country and the country's wealth mainly resides in agriculture About 25\% of Pakistan's agriculture accounts for about $21 \%$ of GDP and employs about $43 \%$ of the labour force. No proper prescription is respected in the country for the purchasing of antibiotics. Some of the most commonly used classes of antibiotics in Pakistan are [6].

a) Penicillins such as penicillin and amoxicillin

b) Cephalosporins such as cephalexin (Keflex)

c) Macrolides such as erythromycin (E-Mycin), clarithromycin (Biaxin), and azithromycin (Zithromax)

d) Fluoroquinolones such as ciprofolxacin (Cipro), levofloxacin (Levaquin), and ofloxacin (Floxin)

e) Sulfonamides such as co-trimoxazole (Bactrim) and trimethoprim (Proloprim)

f) Tetracyclines such as tetracycline (Sumycin, Panmycin) and doxycycline (Vibramycin)

g) Aminoglycosides such as gentamicin (Garamycin) and tobramycin (Tobrex) [22].

Due to the poor economic and lack of basic health facilities in Pakistan. Residents of mainly rural areas find antibiotics easily accessible. Lack of awareness to the damage caused by misusage of such drugs makes them prevalent in the rural parts of the country. The Use of old syringes and expired antibiotics is also common which leads to further diseases than antibiotic resistance [7].

\section{Illiteracy}

Pakistan has a population of about 184 million, with sex ratio of 105.6: 100 . It is estimated that about $62 \%$ of the people are residing in rural and $38 \%$ in urban areas. $42 \%$ population (age $10+$ ) is illiterate. Wide discrepancies persist in education indicators pertaining to provinces/areas, location (urban vs. rural) and gender. At the national level, about two third women of age 15+ cannot read and write, and 35\% girls remain out of school. Gender Parity Index in case of participation in primary education is 0.82 . It is estimated that over 6.7 million children are out of school, and majority of them (62\%) are girls [8] (Figure 2). The literacy rate of Pakistan has been said to drop by $2-3 \%$ by the year 2015 . People have not completely acknowledged the side effects of the taken antibiotics. Fast relive is the only main concern [8].

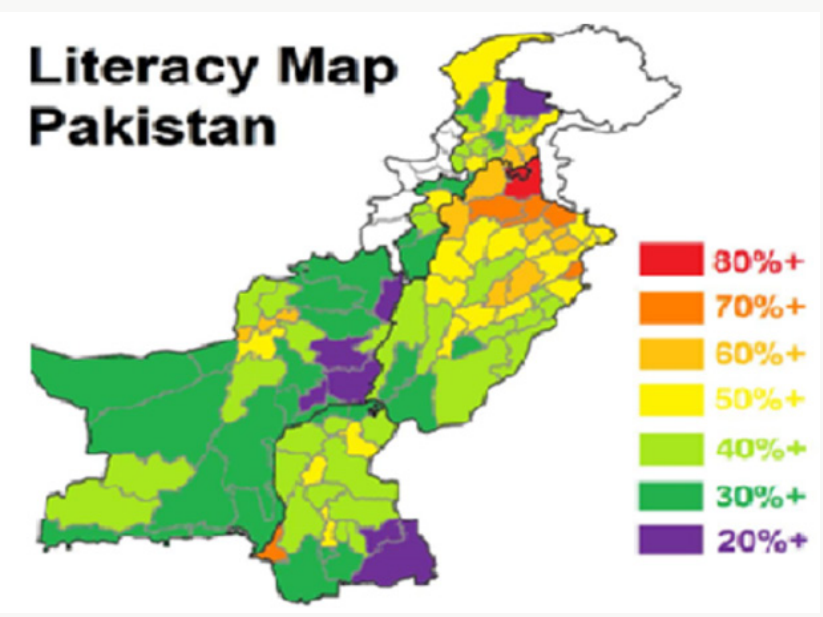

Figure 2: Literacy Map of Pakistan.

\section{Self-Medication and Fake Medical Practitioners}

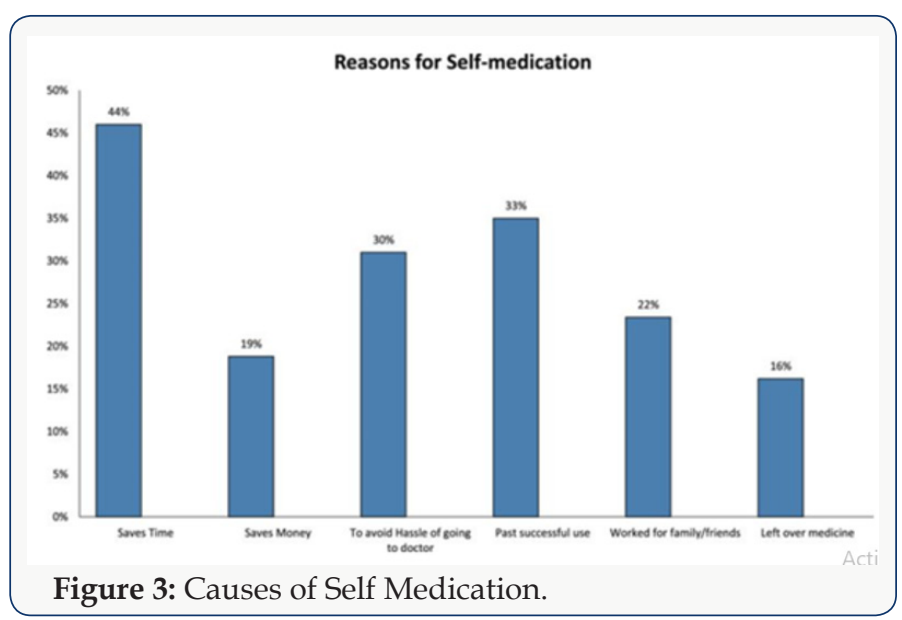

World Health Organization has mentioned, according to a survey that self-medication, if administered appropriately and responsibly can help prevent and treat diseases economically and without medical consultation $[9,10]$. In many developing countries, well-trained health personnel are scarce and cannot serve the entire population, especially in rural areas. Community health workers and others with minimal training treat minor ailments [11]. The Pakistan Medical Association (PMA) released a report stating there are more than 600,000 non-registered Medical Practitioners working in the country. Even Metropolitan cities of Pakistan like Islamabad, Karachi and Lahore are home to self-prescription and non-qualified doctors prescribing. The most common antibiotic used for self-medication purposes was amoxicillin which was used by $81(41.4 \%)$ study participants. Amoxicillin was followed by 
Metronidazole (30.5\%), Ciprofloxacin (12.7\%) and Cotrimoxazole (9.5\%). Erythromycin and Ampicillin/cloxacillin were used by $6.7 \%$ and $6.2 \%$ of the sample population respectively and other antibiotics were amongst the less frequently used antibiotics $[12,5]$ (Figure 3).

\section{Antibiotic Use Other than Humans}

Pakistan is an agricultural country and It is seen in Pakistan that use of antibiotic use is not only restricted to humans only. Sprays of antibiotics used on plants and livestock to increase their mass and yields is increasing the antibiotic resistance among the population. Antibiotics are essential for control of bacterial diseases of plants, especially fire blight of pear and apple and bacterial spot of peach. Streptomycin is used in several countries; the use of oxytetracycline, oxalinic acid and gentamicin is limited to only a few countries. Springtime antibiotic sprays suppress pathogen growth on flowers and leaf surfaces before infection; after infection, antibiotics are ineffective. Antibiotics are applied when disease risk is high but in Pakistan it is used to obtain high yield and faster harvest to sale of fruits and vegetables ignoring the fact that these antibiotics are producing resistant in bodies of those who intake these produce. Livestock are also victims of misuse antibiotics to relieve of even non-serious diseases for fast recovery [13] (Figure 4).

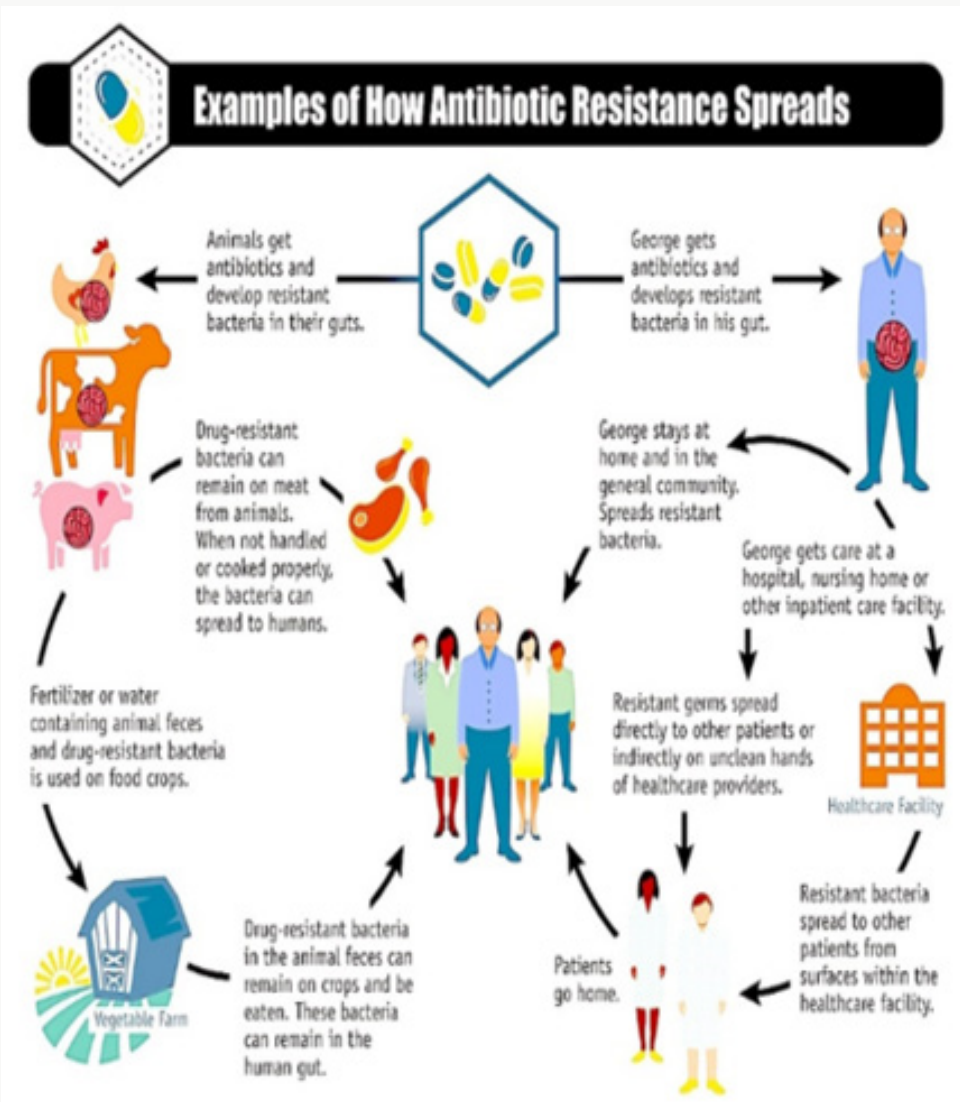

Simply using antibiotics creates resistance. These drugs should only be used to treat infections.

Figure 4: How Antibiotic Resistance Spreads.

\section{Antibiotics Resistance Due to poor quality of Drugs}

It is important to note that poor quality medicine can be critically damaging to public health in many ways beyond promoting anti-microbial resistance. Falsified medicines with no active ingredients leave both chronic and infectious diseases untreated, prolonging illness and the expense of treatment, and sometimes leading to death. For many infectious diseases including HIV and malaria, treatment failure leads to increased pathogen loads and thus increases the chance of infections being passed on. Fake vaccines, for their part, allow a person who would otherwise develop immunity to remain susceptible to infection. Poorly made or mislabelled medicines containing the wrong ingredients can be toxic, again killing the people who take them in the hope of a cure. Medication scares undermine public confidence in the health system, and the massive trade in fake medicine fuels and funds criminal networks while siphoning income away from legitimate business. These issues are, however, beyond the scope of this paper; here we focus only on those issues which affect or are affected by antimicrobial resistance [14].

\section{Causes of Antimicrobial Resistance}

To better appreciate the causes of AMR, we need to understand the various sequential steps involved for a drug to get to a patient 
and the eventual use, which include; production, distribution, prescription, dispensing, and finally consumption of the drug by the patient or use in animal production. Consequently, any imprudent practice along this flow may result in the emergence of resistance [15].

\section{Drug dispensers and drug quality}

the lack of appropriate regulations in the sales of antimicrobials is also a driving factor in the access and misuse of antimicrobials. In most developing countries, antimicrobials can be purchased without medical prescription and are usually dispensed on the streets by untrained persons. These drug vendors will sell medications just to make a sale and accommodate patients' ability to pay. Even pharmacies operating without a license, appear to be more accessible to the public as they have shorter waiting time, do not charge consultation fees and above all are willing to negotiate treatment options to adjust to the financial ability of the patients. Retail pharmacies in developing countries especially in Pakistan have emerged as the primary level of outpatient care rendering unauthorized services from consultation, diagnosis, prescription and dispensing of medication. It has also been shown that many antimicrobials dispensed in Pakistan are of questionable pharmacological quality. Adverse climatic conditions such as high ambient temperatures and humidity may affect the overall quality of the antimicrobials during storage. Poor storage also increases the risk of degradation of the drug. Degraded medicines contain less than stated dose, implying that patients consume less than optimal dose of the drug.

There is also a problem of outright counterfeit, in which the drug may contain little or no active substance of the antimicrobial or the wrong substance. The influx of counterfeit and sub-standard antimicrobials into the pharmaceutical markets in some regions is a major problem as these preparations of reduced potency also result in pathogens being exposed to sub-therapeutic concentrations of the drug. A study revealed that, out of 284 antimalarial obtained from 132 vendors, $32 \%$ of chloroquine, $10 \%$ quinine, and $13 \%$ sulfadoxine/pyrimethamine were likely to be fake. In addition, some of the quinine contained chloroquine while some chloroquine contained no active ingredient or an amount lower than the expected concentration [15] (Tables $1 \& 2$ ).

Table 1: Factors and stakeholders contributing to the problem of antimicrobial resistance.

\begin{tabular}{|c|c|c|}
\hline Factors & Contribution & Example \\
\hline $\begin{array}{l}\text { Poor drug } \\
\text { quality }\end{array}$ & $\begin{array}{l}\text { Sales of counterfeit adulterated and poor quality antibi- } \\
\text { otics }\end{array}$ & $\begin{array}{l}\text { These poor quality antibiotics can produce sub-inhibitory concentra- } \\
\text { tion in vivo, which increases the selection of resistant strains }\end{array}$ \\
\hline Regulators & $\begin{array}{c}\text { While most developed countries have developed AMR } \\
\text { action plans, this is still lacking in many developing coun- } \\
\text { tries especially in Africa }\end{array}$ & $\begin{array}{l}\text { Most countries lack the resources to enforce policies regarding the } \\
\text { manufacture and distribution of sub-standard drugs }\end{array}$ \\
\hline Prescribers & $\begin{array}{l}\text { Excessive clinical use and misuse is partially responsible } \\
\text { for increase rate of resistance }\end{array}$ & $\begin{array}{l}\text { Variation in prescription practice among health care provider. Some- } \\
\text { times there is prescription of a wrong drug, wrong doses, or antimicro- } \\
\text { bial not necessary at }\end{array}$ \\
\hline Dispensers & $\begin{array}{l}\text { Drug vendors usually have little or no knowledge of the } \\
\text { required dosage regimen, indication, or contraindications }\end{array}$ & $\begin{array}{l}\text { Medications are usually purchased in small aliquots from roadside } \\
\text { stall and storage and distribution is usually done under inadequate } \\
\text { conditions }\end{array}$ \\
\hline Users (patients) & $\begin{array}{l}\text { High rate of self- medication and lack of treatment com- } \\
\text { pliance }\end{array}$ & $\begin{array}{l}\text { Patients fail to adhere to dosage regimens and discontinue treatment } \\
\text { when symptoms subside }\end{array}$ \\
\hline
\end{tabular}

Table 2: Factors that contribute to poor-quality drugs.

Factor(s) contributing to poor-quality drugs Substandard drugs:

\begin{tabular}{|c|r|}
\hline 1. & Reduced stability of drugs in developing countries due to environmental conditions and poor storage \\
\hline 2. & No good manufacturing process in developing countries \\
\hline 3. & Poor quality control during manufacture \\
\hline 4. & Poor surveillance about expiration dates and storage conditions in poor settings \\
\hline 5. & Use of non-standardized pharmacopoeias by many developing countries \\
\hline 6. & Counterfeit drugs \\
\hline 7. & Financial interests: crime, corruption of politicians and industry \\
\hline 8. & High demand for antimicrobials and vaccines exceeds supply \\
\hline 9. & High prices of original drugs. \\
\hline 10. & stakeholders \\
\hline
\end{tabular}




\begin{tabular}{|c|c|}
\hline 11. & Development of Internet \\
\hline 12. & High rate of illiteracy and very low income of population in less-developed countries. \\
\hline 13. & $\begin{array}{c}\text { Lack of sensitization of people in less-developed countries to the impact and dangers of counterfeit drugs purchased from unauthorized sales- } \\
\text { men }\end{array}$ \\
\hline
\end{tabular}

\section{Environmental and Hygenic Factors}

Antimicrobial resistance (AMR or AR) is the ability of a microbe to resist the effects of medication that once could successfully treat the microbe [16-18]. The term antibiotic resistance (AR or ABR) is a subset of AMR, as it applies only to bacteria becoming resistant to antibiotics. Resistant microbes are more difficult to treat, requiring alternative medications or higher doses of antimicrobials. These approaches may be more expensive, more toxic or both. Microbes resistant to multiple antimicrobials are called multidrug resistant (MDR). Those considered extensively drug resistant (XDR) or totally drug resistant (TDR) are sometimes called "superbugs" [19].

\section{Veterinary Medicine}

Main article: Antibiotic use in livestock, Infographics showing antibiotic resistance from the farm to the table

All animals carry bacteria in their intestines. Antibiotics are given to animals. Antibiotics kill most bacteria. Butresistant bacteria survive and multiply. The World Health Organization concluded that inappropriate use of antibiotics in animal husbandry is an underlying contributor to the emergence and spread of antibioticresistant germs, and that the use of antibiotics as growth promoters in animal feeds should be restricted [20]. The World Organisation for Animal Health has added to the Terrestrial Animal Health Code a series of guidelines with recommendations to its members for the creation and harmonization of national antimicrobial resistance surveillance and monitoring programs, monitoring of the quantities of antibiotics used in animal husbandry [21], and recommendations to ensure the proper and prudent use of antibiotic substances. Another guideline is to implement methodologies that help to establish associated risk factors and assess the risk of antibiotic resistance [22].

\section{Water, Sanitation, Hygiene}

Infectious disease control through improved water, sanitation and hygiene (WASH) infrastructure needs to be placed at the center of the antimicrobial resistance (AMR) agenda. The spread of infectious diseases caused by inadequate WASH standards is a major driver of antibiotic demand in developing countries [23]. Growing usage of antibiotics together with persistent infectious disease levels have led to a dangerous cycle in which reliance on antimicrobials increases while the efficacy of drugs diminishes [23]. The proper use of infrastructure for water, sanitation and hygiene (WASH) can result in a 47-72 percent decrease of diarrhea cases treated with antibiotics depending on the type of intervention and its effectiveness [23]. A reduction of the diarrhea disease burden through improved infrastructure would result in large decreases in the number of diarrhea cases treated with antibiotics. Sanitation and water for all by 2030 is Goal Number 6 of the Sustainable Development Goals.

\section{Water Pollution}

An increase in hand washing compliance by hospital staff results in decreased rates of resistant organisms. Antibiotic resistance is a growing problem among humans and wildlife in terrestrial or aquatic environments. In this respect, the spread and contamination of the environment, especially through water pollution "hot spots" such as hospital wastewater and untreated urban wastewater, is a growing and serious public health problem $[24,25]$. Antibiotics have been polluting the environment since their introduction through human waste (medication, farming), animals, and the pharmaceutical industry [26]. The contribution of the pharmaceutical industry is so significant that parallels can be drawn between countries with highest rate of increasing antibiotic resistance and countries with largest footprint of pharmaceutical industry. As bacteria replicate quickly, the resistant bacteria that enter water bodies through wastewater replicate their resistance genes as they continue to divide. In addition, bacteria carrying resistance genes have the ability to spread those genes to other species via horizontal gene transfer. Therefore, even if the specific antibiotic is no longer introduced into the environment, antibioticresistance genes will persist through the bacteria that have since replicated without continuous exposure. Antibiotic resistance is wide spread in marine vetebrates and they may be important reservoirs of antibiotic resistant bacteria in marine environment [27].

\section{Prevention}

Important strategies needed for minimizing antibiotic resistance are as follows [28].

a) Antibiotic stewardship to maintain the value of existing and future antibiotics

b) The timing of prescription to use the effective antibiotics sooner rather than later

c) To develop and approve ten new antibiotics by 2020 Development of a molecular method for detecting antibiotic resistance genes (Figures 5 \& 6). 


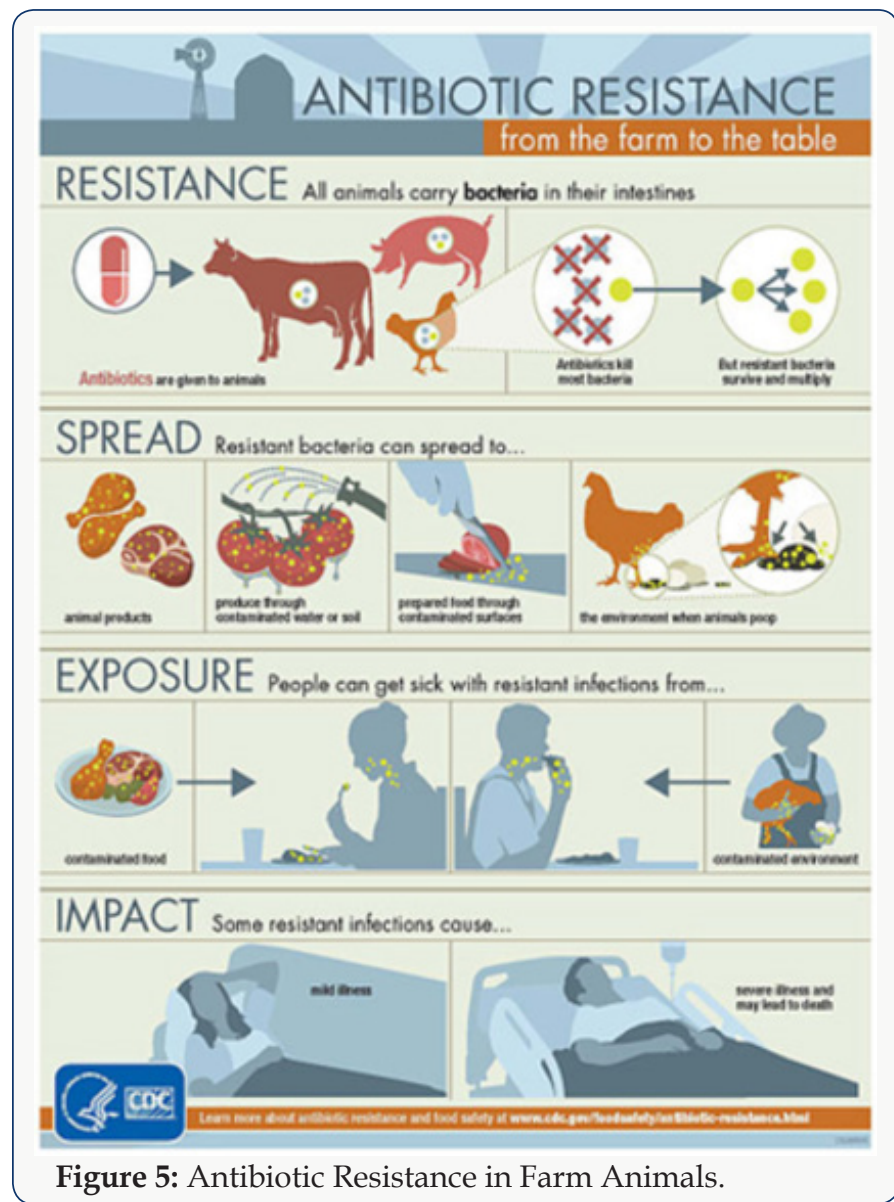

I single antibiotic $\quad$ more than 1 antibiotics
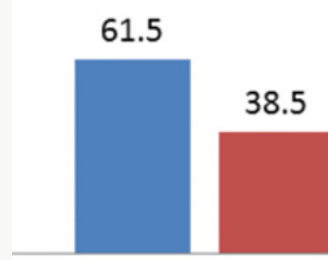

patient demand

pharmacist recommendation

Figure 6: Antibiotics dispensed in case of self-medication.

\section{Transmission of Antibiotic Resistance}

It has been noted and observed by many experts that overuse and misuse of antibiotics has led to resistance all over the world specially in the developing countries like Pakistan. Due to this reason most of the patients are not getting cured by previously used antibiotics. While addressing a National antimicrobial resistance (AMR) organized by AKU. It was said by Dr Sadia Shakoor (assistant professor of pathology and laboratory medicine at AKU). "Antibiotic resistance is a much problem in Pakistan as in the western hemisphere" [2]. This resistance is due to the change in nature of the bacteria and becomes severe due to several multiplications of bacterial generations which cause destruction in human body [1]. Another most common cause of antibiotic resistance in Pakistan is that the patients get antibiotics from medical stores without the physicians description [2]. Multidrug resistance (MDR) is increasing day by day in Pakistan [1].

\section{Prodrug Resistant Bacteria}

Many PDR microorganisms have been identified in Pakistan. These organisms include Pseudomonas, Enterobacter, Enterococcus, Acinetobacter and E coli. Resistant staphylococcus aureus has also been treated as PDR. After the discovery of PDR bacteria TDR bacteria have also evolved [18].

\section{Cause of Infections in New Borns}

Seventy one percent of the new born infections in Pakistan are a result of Antibiotic Resistant Bacteria (ARB) control [29]: A step should be taken in order to prevent medical store employees to give antibiotics without the physician's prescription [2]. Fearing widespread deaths because of PDR and TDR bacteria, leading Pakistani surgeons to form the Surgical Infection Control Society $f$ Pakistan and prepared a set of guidelines for surgeons all over the country to deal with the phenomenon [13]. Elimination of overdose of antibiotics should be a major priority in every country [30].

\section{Conclusion}

In Pakistan; resistance against frequently used antibiotics is increasing day by day in Pakistan like other under developed countries ( $3^{\text {rd }}$ world countries). Community educational programs must be carried out for general population as well as for health care professionals. A revised line of management should be developed locally in accordance with the susceptibility pattern of pathogens to avoid further resistance as well as morbidity of the patient. Antibiotic susceptibility studies should be carried out in each hospital situated at different geographical locations of the country to develop local susceptibility profile against each bacterium circulating in that area.

\section{References}

1. (2017) Muhammad Sohail Afal: Emergance of antibiotic resistance in Pakistan, a clear problem for future. Journal of Vaccines and vaccination 8(6): 375 .

2. (2018) Expert advice on combating antimicrobial resistance in pakistan.

3. (2018) Study finds alarming rate of antibiotic resistance in Pakistan.

4. (2018) Sharprise in antibiotic use in Pakistan.

5. Syed Jawad Shah, Hamna Ahmad, Rija Binte Rehan, Sidra Najeeb, Mirrah Mumtaz (2014) Self-medication with antibiotics among nonmedical university students of Karachi: a cross-sectional study. BMC Pharmacology and Toxicology 15: 74.

6. (2017) Types of antibiotics.

7. Okeke IN, Klugman KP, Bhutta ZA, Duse AG, Jenkins P (2005) Antimicrobial resistance in developing countries part 2 stratigies for containment. The Lancet 5(9): 568-580.

8. Allah Bakhsh Malik (2015) UNESCO Confucius Laureate Additional Secretary, Ministry of Education, Trainings and Standards in Higher Education \& Director General, Academy of Educational Planning and Management, Islamabad Mr. Nasir Amin In-charge National Education Management Information System/Senior System Analyst Academy of 
Educational Planning and Management, Islamabad Ms. Khalida Ahmad Education Specialist, UNICEF, Islamabad Dr. Eshya Mujahid Mukhtar National Consultant, Islamabad Dr. Muhammad Saleem National Consultant, Islamabad Mr. Muhammad Bilal Kakli Programmer, National Education Management Information System, Academy of Educational Planning and Management, Islamabad: Pakistan Education for All Review Report.

9. KS Kiyingi, JAK Lauwo (1993) World Health Forum. Drugs in the Home: Danger and Waste. Edited by WHF 14(4): 381-384.

10. (1998) The role of pharmacist in self care and medication.

11. Okeke IN, Lamikanra A, Edelman R (1999) Socioeconomic and behavioral factors leading to acquired bacterial resistance to antibiotics in developing countries. Emerg Infect Dis 5(1): 18-27.

12. Shankar P, Partha P, Shenoy N (2002) Self-medication and non-doctor prescription practices in Pokhara valley, Western Nepal: a questionnairebased study. BMC Family Practice 3: 17.

13. (2018) Emergance of drug resistant bacteria in Pakistan worries doctors.

14. (2015) Essential Medicines and Health Products Information Portal.

15. Theodoros Kelesidisa, Matthew E Falagas (2015) Substandard/ Counterfeit Antimicrobial Drugs. PMC 28(2): 443-464.

16. (2016) Review on Antimicrobial Resistance.

17. (2015) Antimicrobial resistance Fact sheet.

18. (2017) About Antimicrobial Resistance-Antibiotic/Antimicrobial Resistance-CDC.

19. (2009) Antibiotic Resistance Questions \& Answers. Get Smart: Know When Antibiotics Work. Centers for Disease Control and Prevention, USA.
20. Anitibiotic Resistance (2017) World Heath Organization.

21. OIE - World Organisation For Animal Health.

22. OIE - World Organisation for Animal Health: Review on Antimicrobial Resistance.

23. Araya P, Julia Hug, Genevieve Joy, Felicia Oschmann, Susana Rubinstein (2016) The Impact of Water and Sanitation on Diarrhoeal Diseases Burden and Over Consumption of Antibiotic.

24. Yezli S, Li H (2012) Antibiotic resistance amongst health care-assosiated pathogens in China. International Journel of antimicrobial agents 40(5): 389-397.

25. Marti E, Variatza E, Balcazar JL (2014) The role of aquatic ecosystems as reservoirs of ntibiotic resistance. Trends in Microbiology 22(1): 36-41.

26. Martinez JL, Olivares J (2012) Environmental Pollution by Antibiotic Resistance Genes. In PL Keen, MH Montforts, Antimicrobial Resistance in the Environment. Hoboken, NJ (Eds.): John Wiley \& Sons:Review on Antimicrobial Resistance. Wiley \& Sons, pp. 151-171.

27. (2016) Bigggest Threats-Antibiotics/Antimicrobial resistance.

28. (2017) HealthMap Resistance.

29. (2018) Antibiotic resistant bacteria responsible for 71 newborn infections in Pakistan. Antibiotic-resistant-bacteria-responsible-for-71newborn-infections-in-Pakistan.

30. (2018) Pakistan Ranked Among Countries Which Use the Most Antibiotics.

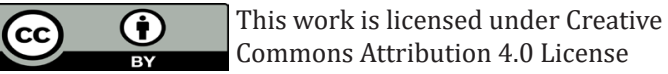

To Submit Your Article Click Here:

Submit Article
DOI: $10.32474 /$ CTBM.2018.01.000101

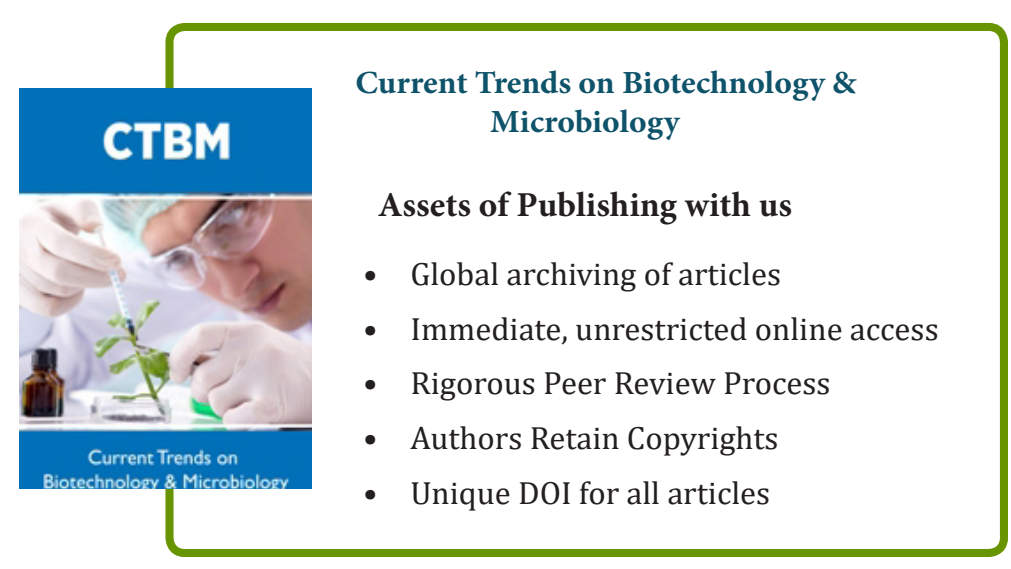

